\title{
Orthopaedic Surgeons and Smartphones
}

\author{
Ahmed M. Ahmed ${ }^{1} \quad$ Ahmed A. Khalifa ${ }^{1, \odot}$ \\ ${ }^{1}$ Department of Orthopaedic and Traumatology, Qena Faculty of \\ Medicine and Its University Hospital, South Valley University, \\ Qena, Egypt
}

\begin{abstract}
Address for correspondence Ahmed M. Ahmed, MBBCh, Department of Orthopaedic and Traumatology, Qena Faculty of Medicine and Its University Hospital, South Valley University, Kilo 6 Qena-Safaga highway, South Valley University, Qena 83523, Egypt (e-mail: drahmedabdou1993@gmail.com).
\end{abstract}
Abstract
Keywords
- orthopaedic
- relationship
- smartphones
- surgeon

Phone technology has witnessed revolutionary advancement in the form of smartphones. Health care professionals, especially orthopaedic surgeons, have been able to make the best use of such remarkable devices. Surveys conducted over years in many different countries have shown how often orthopaedic personnel use smartphone apps for both work and education. Moreover, phone companies have provided surgeons with many useful apps that can be used in the operating theater and emergency room as well. However, there are some concerns regarding the validity of information available in the applications.

\section{Introduction}

A smartphone is defined as a cellular telephone designed with an integrated computer which can execute a wide variety of tasks, including running various applications (apps) that are not typically found on a traditional cell phone. It is known by its manufacturer or operating system (OS), and the current leading systems are Blackberry, iPhone, and Android platforms. Among the most rapidly growing categories of programs, the medical apps are applications which include many programs designed specifically for orthopaedic surgeons assisting them not only in education but also in their surgical practice. ${ }^{1,2}$

\section{How Often Do Orthopaedic Surgeons Use Smartphones?}

In a survey, conducted in 2011, Franko ${ }^{3}$ found that more than half of the participants used smartphone apps in their clinical practice. Almost all of them were ready to pay an average of nearly $\$ 30$ for an app that saves 5 to 10 minutes a day or 30 minutes per week. The most popular apps were textbooks, references, techniques, board reviews, and billing. Epocrates (Epocrates Inc), a free drug reference app, was mentioned by a huge number of participants as their most useful app, while Medscape (WebMD LLC), a drug and procedure reference, as their second most useful one. ${ }^{1,2}$ Another survey, performed in Australia by James

Aahmed A. Khalifa's ORCID is https://orcid.org/0000-0002-0710-6487.
Churchill ${ }^{4}$ in 2012, revealed that all but two reported using their phones for work on a regular basis. The survey results showed that $79 \%$ of the surgeons used mobile technology primarily for professional contact, and nearly $65 \%$ relied on online educational resources. The ability to view X-rays and other radiology imaging on their devices was the most desired future feature. Most orthopaedic caregivers who responded to the survey confirmed that the most fundamental role played by mobile phones is to access patient investigations and past records.

\section{Should We Be Concerned about Using Smartphones?}

Utility of smartphone apps rests in novelty, accessibility, and portability. ${ }^{3}$ Furthermore, smartphone-assisted surgery has proven to be advantageous in many ways, such as accurate digital angle measurements, not time consuming, no extra learning curve, not space occupying, and no radiation. ${ }^{5}$ However, the idea of using smartphone apps in clinical practice is not without concern. Currently, there is no way to regulate the content or validity of information in released programs. ${ }^{6}$ Besides, consumers either must independently verify the information provided or must trust the name of the app publisher. There are also relevant concerns regarding the lack of medical professional involvement in app design. ${ }^{7.8}$ While U.S. Food and Drug Administration (FDA) has lied down regulations regarding

\section{received}

April 15, 2019

accepted

April 27, 2019
DOI https://doi.org/

10.1055/s-0039-1692599

ISSN 2455-7420.
(C2019 Medical and Surgical Update Society
License terms

(ㅇ) (1) $\odot \circledast$ 
medical smartphone apps, which nowadays have a significant impact on patient treatment, most medical smartphone apps do not undergo a formal evaluation under the current guidelines. ${ }^{9}$ Another disadvantage of using smartphones intraoperatively is that the sterility may be compromised. ${ }^{5}$

\section{Examples of Apps Available for Orthopaedic Surgeons}

Although some articles state that few apps are designed for orthopaedic surgeons, ${ }^{3,10}$ Rohman and Boddice ${ }^{11}$ suggested many apps that can be of great value for orthopaedic trainee and specialists. Some examples include Netter's Atlas of Human Anatomy, Pastest, AO surgery reference, Campbell's Operative Orthopaedics and Orthobullets. These apps are highly beneficial from an educational point of view.

Furthermore, numerous apps have been developed to be used perioperatively. For instance, the accelerometer of the iPhone has been used to develop a new system to improve acetabular cup orientation in total hip arthroplasty. Peters et $\mathrm{al}^{5}$ used the Angle app (Smudge Apps) to measure inclination of the acetabular cup and the Camera Protractor app (YJ Soft) to determine anteversion. These results had a favorable outcome when compared with the literature on acetabular cup placements with both the freehand and computer-assisted surgery (CAS) techniques. ${ }^{12-14}$ Another application worth mentioning is measurement of the knee flexion angle by Jean-Yves Jenny ${ }^{15}$ in total knee arthroplasty. The results showed a good correlation and coherence between navigated and smartphone measurements, and there was a good intra- and interobserver reproducibility of the smartphone measurements. Another surgical subspecialty that made the best use of smartphones is foot and ankle surgery where Hallux Angles app (Ockendon.net, 2010, WGHealthcare, Letchworth Garden City, United Kingdom) and iPinPoint (i-smartsolutions, 2010, available at www.i-smartsolutions.com) represent a useful alternative to measure the hallux valgus angle (HVA) and intermetatarsal angle (IMA), respectively.

Smartphones are beneficial not only in the operative theater but also in the emergency room. Now they are preferred over the traditional hospital pager systems which are no longer considered to be efficient. ${ }^{16}$ The literature suggests that photographic images of the injured extremities, computed tomography, and magnetic resonance images in video format and X-ray images of extremities can be sent to the orthopaedic consultants using WhatsApp application (WhatsApp Inc.). Therefore, orthopaedic consultants will be able to assess all the patient data wherever they are and deliver to the emergency physicians the medical decisions about the patient without needing to reach the emergency department in a short time. ${ }^{17}$

\section{Conclusion}

Smartphones are becoming more popular among medical personnel. Their apps are now considered indispensable for orthopaedic surgeons. Despite concerns about the reliability of their content, they have proven useful for both educational and surgical purposes.

\section{Conflict of Interest}

None declared.

\section{References}

1 Oehler RL, Smith K, Toney JF. Infectious diseases resources for the iPhone. Clin Infect Dis 2010;50(9):1268-1274

2 Terry M. Medical apps for smartphones. Telemed J E Health 2010;16(1):17-22

3 Franko OI. Smartphone apps for orthopaedic surgeons. Clin Orthop Relat Res 2011;469(7):2042-2048

4 Churchill J. Mobile technology usage by orthopaedic surgeons and trainees in Australia. J Mob Technol Med 2012;1(2):11-15

5 Peters FM, Greeff R, Goldstein N, Frey CT. Improving acetabular cup orientation in total hip arthroplasty by using smartphone technology. J Arthroplasty 2012;27(7):1324-1330

6 Starman JS, Gettys FK, Capo JA, Fleischli JE, Norton HJ, Karunakar MA. Quality and content of Internet-based information for ten common orthopaedic sports medicine diagnoses. J Bone Joint Surg Am 2010;92(7):1612-1618

7 O'Neill S, Brady RR. Colorectal smartphone apps: opportunities and risks. Colorectal Dis 2012;14(9):e530-e534

8 Hamilton AD, Brady RR. Medical professional involvement in smartphone 'apps' in dermatology. Br J Dermatol 2012;167(1): 220-221

9 Barton AJ. The regulation of mobile health applications. BMC Med 2012;10:46

10 Dala-Ali BM, Lloyd MA, Al-Abed Y. The uses of the iPhone for surgeons. Surgeon 2011;9(1):44-48

11 Rohman L, Boddice T. Letter To The Editor: Smartphone Apps for orthopaedic surgeons. Clin Orthop Rela Res 2013;471(3): 1056:1057

12 Hohmann E, Bryant A, Tetsworth K. A comparison between imageless navigated and manual freehand technique acetabular cup placement in total hip arthroplasty. J Arthroplasty 2011;26(7):1078-1082

13 Gandhi R, Marchie A, Farrokhyar F, Mahomed N. Computer navigation in total hip replacement: a meta-analysis. Int Orthop 2009;33(3):593-597

14 Zheng G, Zhang X. Computer assisted determination of acetabular cup orientation using 2D-3D image registration. Int J CARS 2010;5(5):437-447

15 Jenny JY. Measurement of the knee flexion angle with a Smartphone-application is precise and accurate. J Arthroplasty 2013;28(5):784-787

16 Ellanti P, Moriarty A, Coughlan F, McCarthy T. The use of WhatsApp smartphone messaging improves communication efficiency within an orthopaedic surgery team. Cureus 2017;9(2):e1040

17 Gulacti U, Lok U, Çelik M. Use of WhatsApp application for orthopedic consultations in the ED. Am J Emerg Med 2016;34(7):1305-1307 